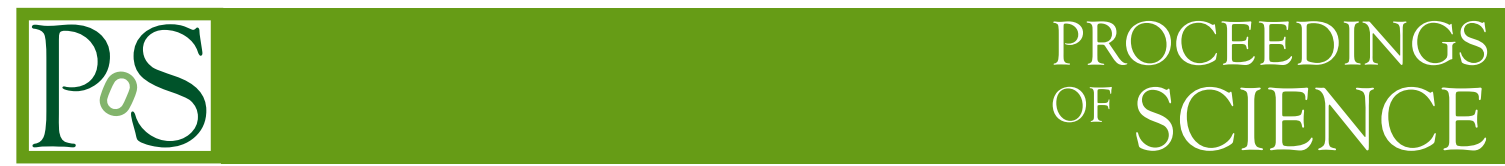

\title{
Design and Performance of ECC-MECC
}

\author{
LUCA SCOTTO LAVINA* \\ INFN-Napoli \\ E-mail: scottoena.infn.it
}

The concept of the Magnetized Emulsion Cloud Chamber is here described. It is shown how the presence of a magnetic field and the use of an emulsion spectrometer permit very good charge reconstruction and enhance the momentum determination capabilities, keeping at the same time the high accuracy and compactness of the Emulsion Cloud Chamber, presently used in the OPERA experiment. The design of such a detector and its tuning, performed so far by means of simulations and a dedicated test beam, is done with the ambitious aim to fulfill all the requirements for an ideal detector operating at a neutrino facility.

10th International Workshop on Neutrino Factories, Super beams and Beta beams June 30 - July 52008

Valencia, Spain

\footnotetext{
${ }^{*}$ Speaker.
} 


\section{Introduction}

The ideal detector for any neutrino facility should be able to exploit all the oscillation channels that are available thanks to the well know neutrino flux composition. Namely, the oscillations $v_{e} \rightarrow v_{\mu}$ (the so-called golden channel), $v_{e} \rightarrow v_{\tau}$ (the so-called silver channel), $\bar{v}_{\mu} \rightarrow \bar{v}_{e}$ (the socalled platinum channel), $\bar{v}_{\mu} \rightarrow \bar{v}_{\tau}$ when a $\mu^{+}$circulates into the decay ring and their CP conjugates in the case of a $\mu^{-}$. Therefore, the ideal detector should be able to measure the momentum and the charge of the leptons (electrons and muons), to identify the decay topologies of the $\tau$ leptons and to perform a complete and accurate kinematical reconstruction of neutrino events in order to reduce the background contamination.

A detector à la OPERA [1] [2], based on the Emulsion Cloud Chamber (ECC) technique, has been proposed to search for the silver channel at a Neutrino Factory through the direct detection of $\tau$ decay topologies [3] [ [ The main limitation factor of this detector is the possibility of measuring the charge only for muons, by an external magnetic spectrometer. The fraction of the $\tau$ decays which can be exploited is thus given by the muonic decay branching ratio only, about $17 \%$.

In this paper it is described the idea of using an ECC detector placed in a magnetic field. This combination provides a detector with very good charge reconstruction and momentum determination capabilities, keeping at the same time the high accuracy and compactness of an ECC. The aim of the magnetized ECC detector is to exploit all the $\tau$ decay modes and to be able to measure the charge of the particles close to the neutrino interaction, further reducing the background.

The paper is organized as follow: first it is briefly recalled the basic performance of an ECC, then it is discussed the layout of an ECC-based detector to be operated immersed into a magnetic field. Finally are shown the performances of a such detector by means of simulations and of a test beam with an emulsion spectrometer prototype performed in a KEK-PS T1 pion beam [5].

\section{The Emulsion Cloud Chamber}

The ECC is a structure made of a sandwich of passive material plates interspersed with emulsion films. It combines the high-precision tracking capabilities of nuclear emulsions with the large mass achievable by employing metal plates as a target. By assembling a large quantity of ECC modules, it is possible to realize $\mathscr{O}($ kton $)$ fine-grained vertex detector optimized for the study of $v_{\tau}$ appearance, through the direct detection of the $\tau$ 's produced in $v_{\tau}$ charged current interactions. It has been adopted by the OPERA Collaboration for a long-baseline search of $v_{\mu} \rightarrow v_{\tau}$ oscillations at the CNGS beam.

The basic element of the OPERA ECC is a cell made of a $1 \mathrm{~mm}$ thick lead plate followed by a thin emulsion film, which consists of $44 \mu \mathrm{m}$-thick emulsion layers on either side of a $210 \mu \mathrm{m}$ plastic base [6]. The number of grains of metallic silver induced by ionization by a charged particle (and produced after the chemical development) in each emulsion layer is about 20. This ensures redundancy in the measurement of particle trajectories and allows the measurement of their energy loss that, in the non-relativistic regime, can help to distinguish different particle masses.

Thanks to the dense ECC structure and to the high granularity provided by the nuclear emulsions, the detector is also suited for electron and $\gamma$ detection, with an efficient electron/pion separation [7]. The energy resolution for an electromagnetic shower is about 20\%. By measuring 


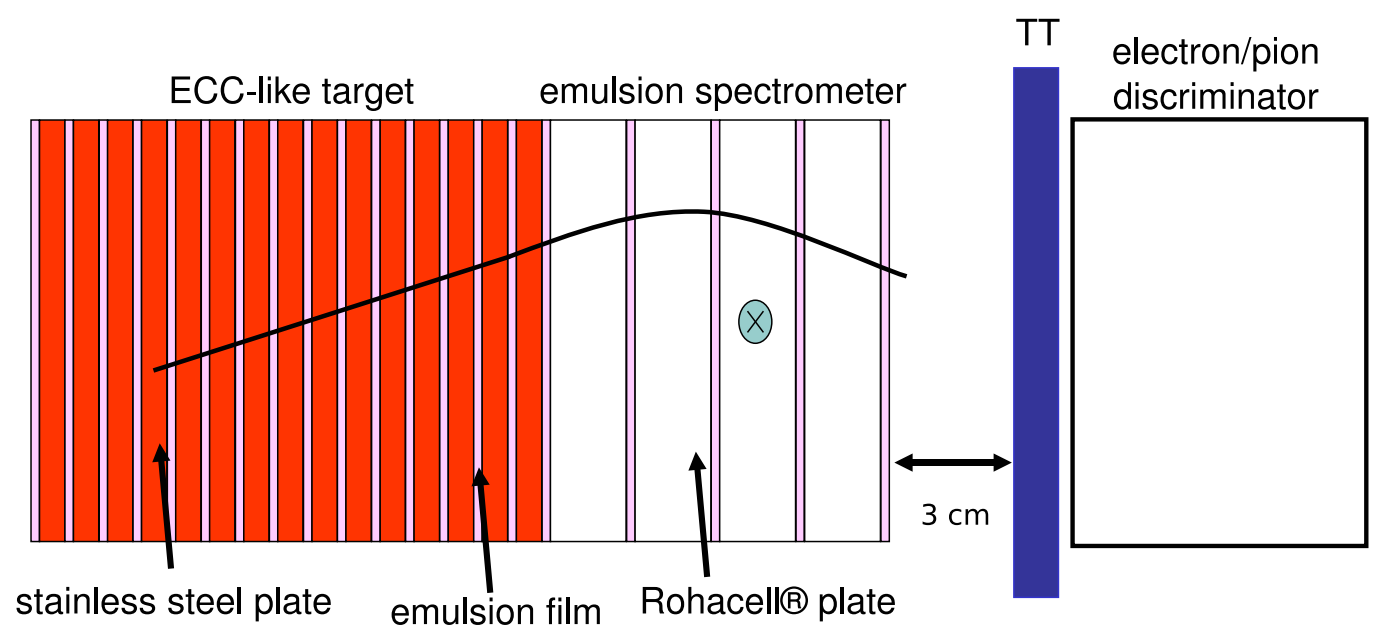

Figure 1: Schematic view of a Magnetized Emulsion Cloud Chamber

the number of grains associated to each track a two-track resolution of $\sim 1 \mu \mathrm{m}$ or even better can be achieved [8]. Therefore, it is possible to disentangle single-electron tracks from electron pairs coming from $\gamma$ conversion in lead. The outstanding space resolution can also be used to measure the angle between subsequent track segments with an accuracy of about $1 \mathrm{mrad}$ [9]. This allows the use of Coulomb scattering to evaluate the particle momentum with a resolution of about $20 \%$ [10] and to reconstruct the kinematical event variables [11].

\section{The Magnetized Emulsion Cloud Chamber}

The Magnetized Emulsion Cloud Chamber (MECC) here envisaged has the modular structure shown in Figure 1 .

The target is a sandwich of passive plates and nuclear emulsions used as tracking devices. The length of the target section in terms of radiation lengths must be sufficiently high to prevent the majority of the electrons to shower before their charge has been measured by the downstream modules. The optimization of the passive material is still undergoing. Here it is presented the stainless steel as a possible choice.

An emulsion spectrometer is located downstream of the target. It consists of a sandwich of nuclear emulsions and very light material called spacer. The function of the spacer is to provide a "long" level arm between two consecutive emulsions (tracking devices) with a stable mechanical structure. A few centimeter thick Rohacell plate fulfills this requirement. The trajectory measured with the emulsions which precede and follow the "spacer" provides the measurement of the charge and momentum of the particle. The target and the spectrometer could mechanically form a single brick of about $10 \mathrm{~cm}$ length.

Downstream of the spectrometer, an electronic target tracker has the aim of providing the time stamp of the events. The scanning of the events is planned to be performed without any electronic detector prediction. Therefore, the time information is mandatory in order to match the emulsion 

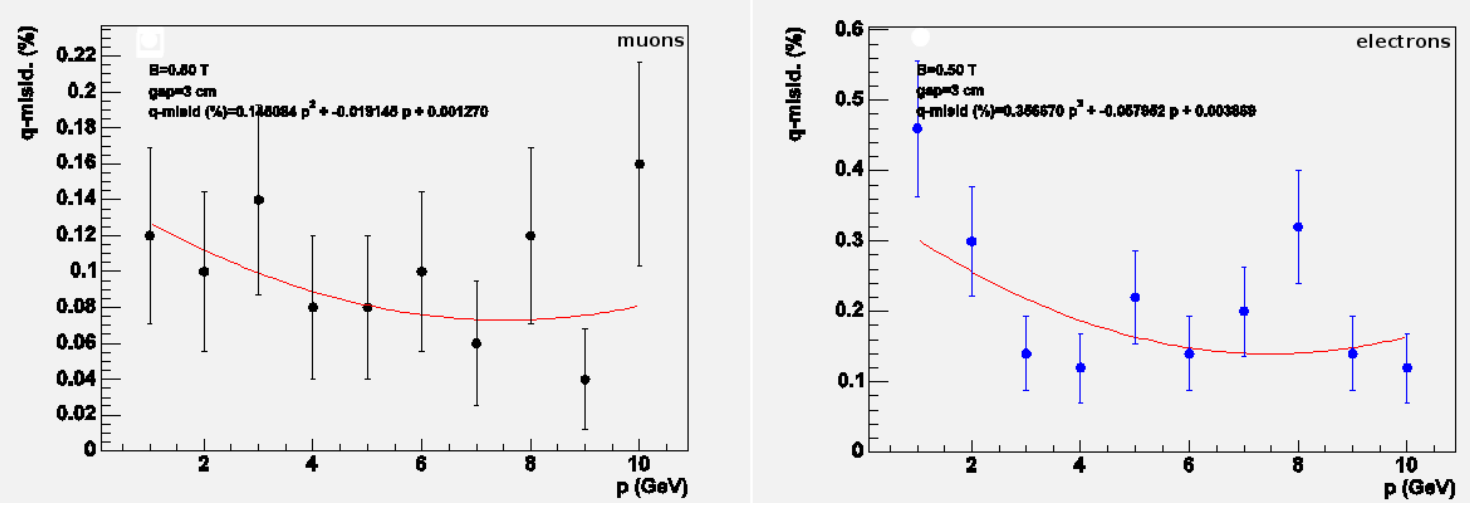

Figure 2: Charge misidentification as a function of the momentum for minimum ionizing particles (left panel) and electrons (right panel), assuming a $3 \mathrm{~cm}$ spacer thickness and a 0.5 Tesla magnetic field.

information with the ones from the electronic detector that allow the charged-current to neutralcurrent event separation.

The most downstream element of the detector is the electron/pion discriminator. Its aim is to provide the electron identification, having already measured the charge and momentum of the primary tracks in the emulsion spectrometer. The combination of a good electron identification with a low pion misidentification probability could be achieved either by a conventional electronic detector or by an emulsion calorimeter (emulsion-lead sandwiches). The choice between the two will be done according to the cost/effectiveness optimization.

The MECC performance both for minimum ionizing particles (MIP) and electrons has been studied by considering different parameters: particle energy in the 1 to $10 \mathrm{GeV}$ range, spacer thickness in the $2-5 \mathrm{~cm}$ range and three values of the magnetic field $(0.25,0.5$ and 1 Tesla). The same nuclear emulsion films as used by OPERA experiment were considered. The thickness of the stainless steel plates has been taken to be $1 \mathrm{~mm}$ with a total of 35 plates (about $2.5 X_{0}$ ). The number of Rohacell spacers is 4 .

Given the target structure described above, the total weight of the target part of each MECC brick is about $3.3 \mathrm{~kg}$. This value has been obtained by assuming for the emulsion sheets the same transverse dimensions as in OPERA $\left(10 \times 12 \mathrm{~cm}^{2}\right)$.

\section{Performances of the MECC}

Monte Carlo simulations have been performed in order to evaluate the momentum resolution and the charge identification efficiency of a MECC. The study was done with a particle energy ranging between 1 and $10 \mathrm{GeV}$ and in function of the detector parameters that have to be optimized: the spacer thickness and the magnetic field intensity. With a spacer thickness of $3 \mathrm{~cm}$ and a magnetic field of 0.5 Tesla, a 30\% (10\%) momentum resolution at 10 (1) $\mathrm{GeV}$ can be achieved. The charge misidentification rate, shown in Figure 2 (left panel), is better than $0.2 \%$ in the considered range. 
The electron momentum and charge measurements are strongly affected by the showering. It has been shown that only $30 \%$ of the electrons with energy in the range 1 to $10 \mathrm{GeV}$ exit the target region without showering. For these events the momentum resolution and the charge identification efficiency, shown in Figure 2 (right panel), are similar to those obtained for muons.

Another important issue is related to the number of interactions that can be stored in a brick preserving the capability of connecting unambiguously the events occurring in the emulsion target with the hits recorded by the electronic detectors. By using a tracker made by $3 \mathrm{~cm}$ strips up to 100 events may be stored into a single brick. This is a very conservative number that insure the capability of the detector to stay on the beam for several years.

A first test of an emulsion spectrometer exposed to a pion beam has been performed in a KEKPS T1 pion beam [5]. It consisted of 2 spacers of $1.5 \mathrm{~cm}$ thickness sandwiched with 3 emulsion films, for a total length of $3 \mathrm{~cm}$. They were located inside a 1 Tesla permanent magnet. The emulsion spectrometer has been exposed to pion beams with momenta $0.5,1$ and $2 \mathrm{GeV}$. The achieved momentum resolution is $\sigma(p) / p \sim(13.3 \pm 0.3) \%$, almost constant in the studied energy range. This result demonstrates the capability of such a detector and also shows that it is possible to study the performance of a MECC in a simple way, given the high modularity of the setup.

\section{Conclusion}

The Magnetized Emulsion Cloud Chamber detector seems to fulfill all the requirements to be a suitable detector for a Neutrino Factory. Indeed, it is able to both detect $\tau$ decays and measure the charge of the electron. Furthermore, it would be also possible to study the golden channel by using an associated electronic detector. The simulations performed so far and the test of an emulsion spectrometer performed in a KEK-PS T1 pion beam gave encouraging results and the physics reach is only limited by the mass required for a MECC based detector.

\section{References}

[1] M. Guler et al., OPERA Proposal CERN/SPSC 2000-028, CERN/SPSC 2001-025, Y. Declais et al., OPERA Proposal CERN/SPSC 2002-029.

[2] R. Acquafredda et al. [OPERA Collaboration], New J. Phys. 8 (2006) 303.

[3] A. Donini, D. Meloni, P. Migliozzi, Nucl. Phys. B 646 (2002) 321 [arXiv:hep-ph/0206034].

[4] D. Autiero et al., Eur. Phys. J. C 33 (2004) 243 [arXiv:hep-ph/0305185].

[5] C. Fukushima et al., Nucl. Instrum. Meth. A 592 (2008) 56.

[6] T. Nakamura et al., Nucl. Instr. Meth. A 556 (2006) 80.

[7] L. Arrabito et al., JINST 2 (2007) P02001 [arXiv:physics/0701192].

[8] T. Toshito et al., Nucl. Instr. Meth. A 516 (2004) 436.

[9] M. De Serio et al., Nucl. Instr. Meth. A 554 (2005) 247.

[10] G. De Lellis et al., Nucl. Instr. Meth. A 512 (2003) 539.

[11] K. Kodama et al., Nucl. Instr. Meth. A 493 (2002) 45. 